\title{
The Heliopause Electrostatic Rapid Transit System (HERTS) - Design, Trades, and Analyses Performed in a Two Year NASA Investigation of Electric Sail Propulsion Systems.
}

\author{
Bruce M. Wiegmann ${ }^{1}$, Todd Scheider ${ }^{2}$, and Andrew Heaton ${ }^{3}$, Jason Vaughn ${ }^{4}$ \\ Marshall Space Flight Center, Huntsville, AL, 35812 \\ Dr. Nobie Stone ${ }^{5}$ \\ NeXolve, Huntsville, AL, 35801 \\ and \\ Dr. Ken Wright ${ }^{6}$ \\ University of Alabama, Huntsville, Huntsville, AL, 35801
}

\section{Introduction}

$\mathrm{P}$

ersonnel from NASA's MSFC have been investigating the feasibility of an advanced propulsion system

known as the Electric Sail (E-Sail) for future scientific exploration missions. This team initially won a NASA Space Technology Mission Directorate (STMD) Phase I NASA Innovative Advanced Concept (NIAC) award and then a two-year follow-on Phase II NIAC award in October 2015. This paper documents the findings from this three-year investigation.

An Electric sail, a propellant-less propulsion system, uses solar wind ions to rapidly travel either to deep space or the inner solar system. Scientific spacecraft could reach Pluto in $\sim 5$ years, or the boundary of the solar system in ten to twelve years compared to the thirty-five plus years the Voyager spacecraft took.

The team's recent focuses have been:

1) Developing a Particle in Cell (PIC) numeric engineering model from MSFC's experimental data on the interaction between simulated solar wind and a charged bare wire that can be applied to a variety of missions,

2) Determining what missions could benefit from this revolutionary propulsion system,

3) Conceptualizing spacecraft designs for various tasks: to reach the solar system's edge, to orbit the sun as Heliophysics sentinels, or to examine a multitude of asteroids.

\section{Introduction}

Harnessing the power of the solar wind, an Electric Sail, or E-sail, is a relatively new concept that promises to deliver high speed propellant-less propulsion. The electric sail is an invention made in 2006 at the Kumpula Space Centre in Finland by Pekka Janhunen. ${ }^{1}$ At its core, an electric sail utilizes multiple positively biased tethers which exchange momentum with solar wind protons via the repelling electric field established around each tether, in other words, by reflecting the solar wind protons. Recognizing the solar wind is a plasma, the effective repelling area of each tether is increased significantly by the formation a plasma sheath around each tether. Figure 1 shows schematically a spacecraft employing an electric sail. The positive voltage bias (>10kV) applied to each tether naturally results in electron collection. Therefore, the electric sail concept necessarily includes an electron source (electron gun) to return collected electrons to space and maintain the positive bias of the tether system.

\footnotetext{
${ }^{1}$ Aerospace Engineer, Advanced Concepts Office, ED04, and AIAA Member.
}

American Institute of Aeronautics and Astronautics 


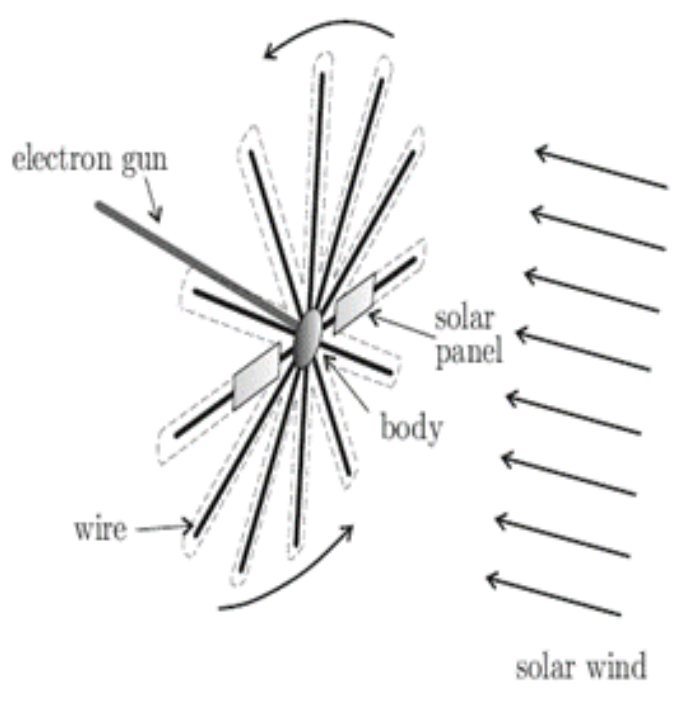

Figure 1. Schematic Diagram of an Electric Sail ${ }^{2}$

Following Janhunen's introduction and development of the electric sail concept, additional work by the Finnish team and others suggested that missions employing electric sails could realize fast trip times to the outer planets and even the edge of the solar system. ${ }^{3,4}$ Interested by such exploration opportunities, the Advanced Concepts Team at NASA's Marshall Space Flight Center (MSFC) began investigating the prospects of using an electric sail to travel to the edge of the solar system (the Heliopause).

In 2014, they received an award from the NASA Innovative Advanced Concepts (NIAC) program to investigate the feasibility of an electric sail mission they developed called the Heliopause Electrostatic Rapid Transit System (HERTS). The HERTS Phase I study concluded an E-sail mission to the Heliopause could be completed within 15 years, which is much faster than any other existing propulsion system could deliver.

While the HERTS Phase I study of an electric sail mission to the Heliopause showed good overall feasibility, some technical challenges remained to be addressed. In 2015, the NIAC program provided $\$ 500 \mathrm{k}$ in resources for a two year Phase II NIAC study with the objective of having the HERTS team investigate some of the key challenges identified in the first study. One of those challenges was: "Lack of a reliable model for solar wind proton and electron interactions with the highly biased wires". ${ }^{5}$ To address this problem, the HERTS team chose to conduct laboratory testing that could be used to anchor a Particle-In-Cell (PIC) spacecraft engineering model capable of reliably extrapolating to solar wind scales. The PIC model and the underlying experimental plasma data recorded in one of MSFC's plasma chambers will be one of this effort's main products. In addition to the PIC model development, analysis of E-Sail mission capture and conceptual designs of notional E-Sail spacecraft are also study products.

\section{General Guidelines}

The results of the Phase I NIAC study Figure 2 found the concept to be feasible from a mission design perspective and identified technical issues for further study. Since most of the E-Sail propulsion system components already have a flight heritage, it can be reasonably expected that a HERTS system capable of reaching the Heliopause in less than 15 years could be developed within a decade and provide meaningful Heliophysics Science in the 2025-2030 timeframe.

American Institute of Aeronautics and Astronautics 


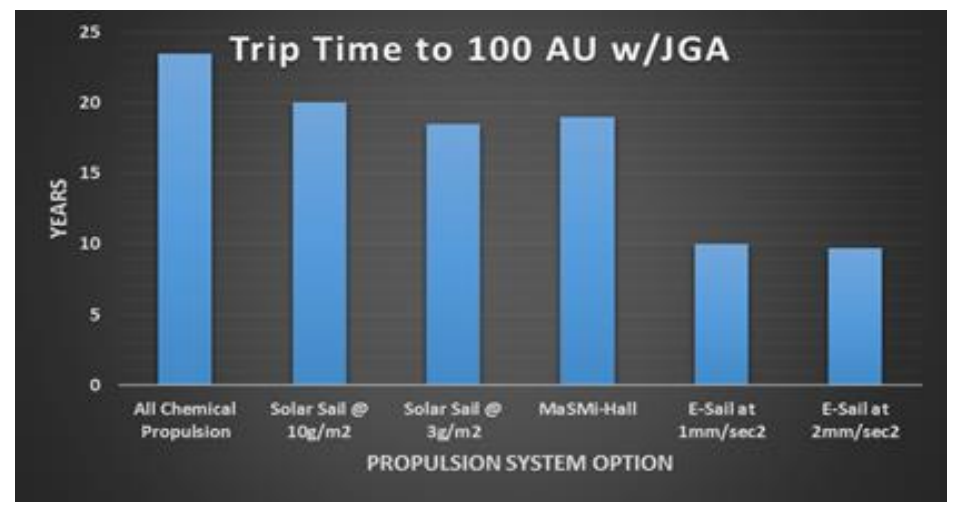

Figure 2. The E-Sail option dramatically reduces trip times to the Heliopause ( $100 \mathrm{AU})$ by $\sim 50 \%$ as compared to other advanced propulsion technologiesFurthermore, the propulsion system can be used to explore any of the major planets or their moons with transit times significantly less than any other concept.

\section{Underlying Physics Behind This Propulsion Scheme}

The interaction of the naturally occurring space plasma with a positively charged conductor in space is very complex. The underlying physics of this interaction as well as the capabilities and limitations of existing space plasma physics models were documented in a paper presented at the 2016 Tethers in Space Conference by one of our team members, Nobie Stone ${ }^{6}$.

In this paper, the set of very complex physics models are reduced to one final set of equations derived from laboratory simulation data. These reduced equations ultimately predict the propulsive force generated by an Electric Sail propulsion system.

The force per unit area (f) exerted by solar wind protons flux per unit area can be calculated from the loss of momentum:

$$
\begin{gathered}
\mathrm{f}=\mathrm{n}_{\mathrm{o}} \mathrm{v}_{\mathrm{o}}\left(\mathrm{M}_{\mathrm{in}}-\mathrm{M}_{\mathrm{out}}\right) \\
\text { where: } \mathrm{f}=\text { force per unit area }\left(\text { NOTE: } 1 / \mathrm{m}^{2}\right) \\
\mathrm{n}_{\mathrm{o}}=\text { ambient plasma density } / \mathrm{m}^{3} \\
\mathrm{v}_{\mathrm{o}}=\text { solar wind drift speed, } \mathrm{km} / \mathrm{sec} \\
\mathrm{M}_{\mathrm{in}} \quad=\text { Momentum into deflection region } \\
\mathrm{M}_{\text {out }}=\text { Momentum out of deflection region } \\
\mathrm{M}_{\mathrm{in}}=\pi / 2\left(\mathrm{~m}_{\mathrm{p}} \mathrm{v}_{\mathrm{o}}\right) \text { and } M_{\text {out }}=m_{p} v_{o} \int_{0}^{\pi / 2} \cos (2 \vartheta) d \vartheta=0 .
\end{gathered}
$$

Then:

$$
\mathrm{f}=\mathrm{n}_{\mathrm{o}} \mathrm{m}_{\mathrm{p}} \mathrm{v}_{\mathrm{o}}^{2}
$$

The propulsive force per unit length $(\mathrm{m})$ of tether $(\mathrm{F})$ is then:

$F=2 r_{s} f$, where $r_{s}$ is determined from Equation 9 of Stone $(2016)^{6}$; shown below:

$$
r_{\mathrm{s}}=3.0 \phi_{\mathrm{w}^{1 / 2}}
$$

where:

$$
\begin{aligned}
& \mathrm{r}_{\mathrm{s}}=\text { the plasma sheath radius for effective proton deflection } \\
& \phi_{\mathrm{w}}=\text { the electrical bias applied to the E-Sail wire array. }
\end{aligned}
$$


For typical solar wind values at $1 \mathrm{AU}$ (i.e., $\mathrm{n}_{\mathrm{o}}=7 \times 10^{6} / \mathrm{m}^{3}, \mathrm{~T}=1.5 \times 10^{5} \mathrm{~K}, \mathrm{v}_{\mathrm{o}}=400 \mathrm{~km} / \mathrm{s}$ ) the force generated per meter of wire at $\phi_{\mathrm{b}}=6 \mathrm{kV}$ is approximately $1.36 \mu \mathrm{N} / \mathrm{m}$. Recent calculations by Janhunen, with $\phi_{\underline{\mathrm{w}}}=20 \mathrm{kV}$, give a force of $0.5 \mu \mathrm{N} / \mathrm{m}$. Using $20 \mathrm{kV}$ here results in $2.49 \mu \mathrm{N} / \mathrm{m}$ - about five times greater than Janhunen's value. We will learn how accurate our estimates and assumptions pertaining to the thrust produced by an electric sail after our NIAC Phase II work is finalized in late September 2017.

\section{Experimental Plasma Testing at MSFC}

\section{A. Testing Framework}

Essential to the operation of an electric sail is the formation of a large plasma sheath around the very small diameter positively biased tether wires. Recall, sheath formation is a fundamental property of plasmas which naturally organize to screen out unbalanced electric fields. The characteristic screening distance is known as the Debye length. As seen in equation (1), the Debye length is a function of the plasma density and electron temperature.

$$
\text { Debye Length }=\lambda_{D}=\sqrt{\frac{\varepsilon_{0} k T_{e}}{q^{2} n_{0}}}
$$

Where $\varepsilon_{0}$ is the permittivity of free space, $k$ is Boltzmann's constant, $T_{e}$ is the electron temperature, $q$ is the electron charge, and $n_{0}$ is the plasma density. In general, the size, or thickness, of a plasma sheath is said to be a few Debye lengths.

Table 1 was created during the test formulation phase to help provide a framework for the laboratory test setup. The test conditions were optimized for scaling where possible, with primary focus on Debye length. It was determined the Debye length of the laboratory plasma:

- Should be much larger than the tether diameter

- Should be much smaller than the plasma test chamber diameter (to avoid wall effects)

The overall test objective, was, for a given set of relevant plasma conditions, measure the extent of the sheath around a biased tether element, and map the deflection of ion trajectories caused by the sheath. Then feed this data into a model capable of extrapolating to E-sail mission scales.

Table 1: Comparison of Parameters: E-sail and Laboratory

\begin{tabular}{|c|l|l|l|}
\hline & Parameter & Value & Comment \\
\hline \multirow{4}{*}{$\begin{array}{c}\text { E-Sail } \\
\text { Mission }\end{array}$} & Proton Speed & $400-450 \mathrm{~km} / \mathrm{s}$ & $\sim 1000 \mathrm{eV}$ \\
\hline & Density & $\sim 5 / \mathrm{cm}^{3}$ & \\
\hline & Electron Temp. & $\sim 12 \mathrm{eV}$ & \\
\cline { 2 - 4 } & Ion Temp. & $\sim 10 \mathrm{eV}$ & \\
\hline & Debye Length & $\sim 10 \mathrm{~m}$ & \\
\hline & Tether Diameter & $\sim 7.5 \times 10^{-5} \mathrm{~m}$ & $\sim 75$ microns \\
\hline & Tether Bias $(+)$ & $>10 \mathrm{kV}$ & \\
\hline \multirow{4}{*}{$\begin{array}{c}\text { Laboratory } \\
\text { Enew } \\
\text { Energy } \\
\text { Analog }\end{array}$} & Ion Speed & $\sim 19-38 \mathrm{~km} / \mathrm{s}$ & $\sim 80-300 \mathrm{eV}$ \\
\hline & Density & $\sim 1 \times 10^{6} / \mathrm{cm}^{3}$ & \\
\hline & Electron Temp. & $<1 \mathrm{eV}$ & \\
\hline & Ion Temp. & $<<1 \mathrm{eV}$ & \\
\hline & Debye Length & $<1 \mathrm{~cm}$ & $>$ Tether Dia. \\
\hline & Tether Diameter & $\sim 1 \mathrm{~mm}$ & \\
\cline { 2 - 4 } & Tether Bias $(+)$ & $100-300 \mathrm{~V}$ & $>$ Ion Energy \\
\hline
\end{tabular}

\section{B. Test Setup}

The Space Environmental Effects Team at NASA's Marshall Space Flight Center was selected to execute the HERTS laboratory investigation, in part, because of their existing operational array of plasma sources, high vacuum test chambers, and diagnostic sensors.

American Institute of Aeronautics and Astronautics 


\section{Plasma Source}

The test team evaluated multiple source options in the context of fitting in the framework described above and, in general, providing a quasi-neutral plasma with drifting ions. Ultimately, a broad-beam gridded ion source, or Kaufman source, was selected. This type of source offers control of the ion drift energy using the accelerating grids. The Kaufman source also provides low energy electrons via its neutralizer cathode. Figure 3 is a picture of the Kaufman plasma source used in the test. Table II provides typical plasma parameters generated by the source.

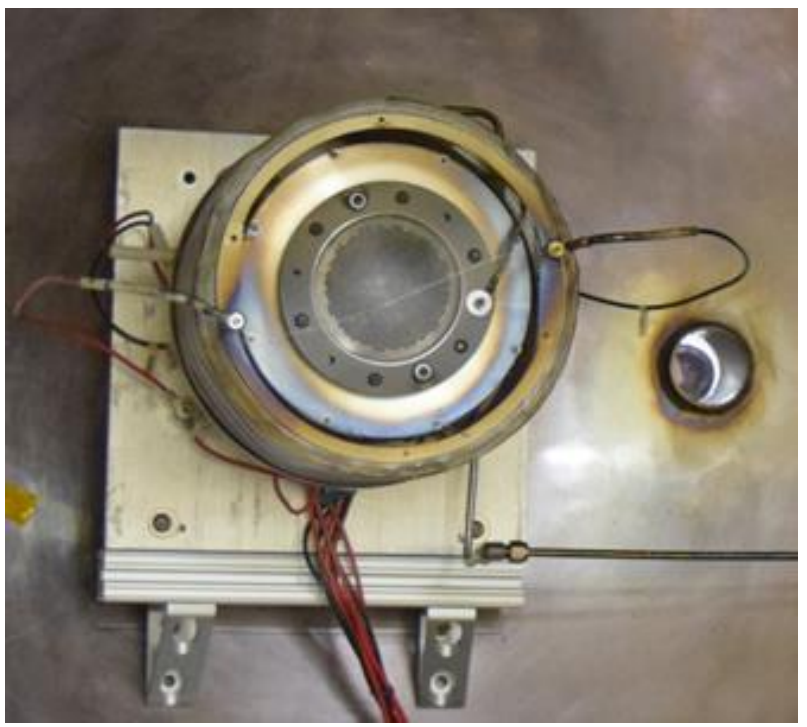

Figure 3. Gridded plasma source (Kaufman type) with neutralizer cathode

All testing was conducted with an Argon plasma. While the Kaufman source is capable of operating with Hydrogen gas, the production of molecular Hydrogen ions $\left(\mathrm{H}_{2}^{+}\right)$along with protons complicates the plasma dynamics and analysis.

\section{Diagnostic Sensors}

Three diagnostic probes were used throughout the test campaign. A spherical Langmuir Probe (LP) measured the plasma electron temperature, plasma density, and plasma potential. The gridded Retarding Potential Analyzer (RPA) provided ion energy and flux. The Differential Ion Flux Probe (DIFP) provided ion trajectory information. ${ }^{7}$ All of the probes were mounted together as a movable array. Figure 4 shows the probe arrangement.

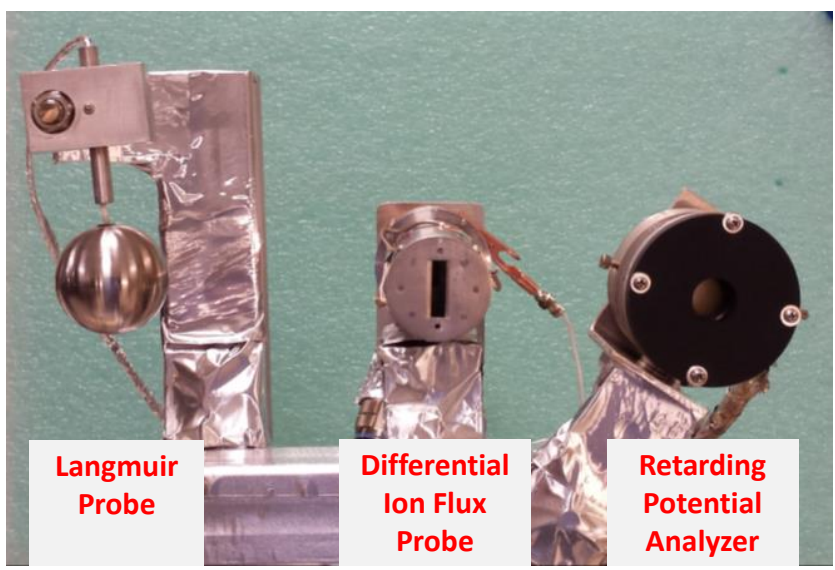

Figure 4. Plasma diagnostic probe array 
The LP and RPA are common diagnostic probes for making plasma measurements, however the DIFP is a unique instrument, and given its important role in the testing, merits further description. A cross-section schematic of the DIFP is shown in Figure 5.

The DIFP uses electrostatic deflection to steer ions into a collector where they are measured as current. The level 1 output of the DIFP is current versus deflector voltage. By calibrating the DIFP at known angles, the product of the DIFP can be transformed into flux versus angle, i.e. the trajectory of ions beam can be determined. As conceived by Stone, the DIFP instrument can yield ion angle and energy information. Since a dedicated RPA was part of the instrument array, the energy measurement feature of the DIFP was not employed for the test campaign.

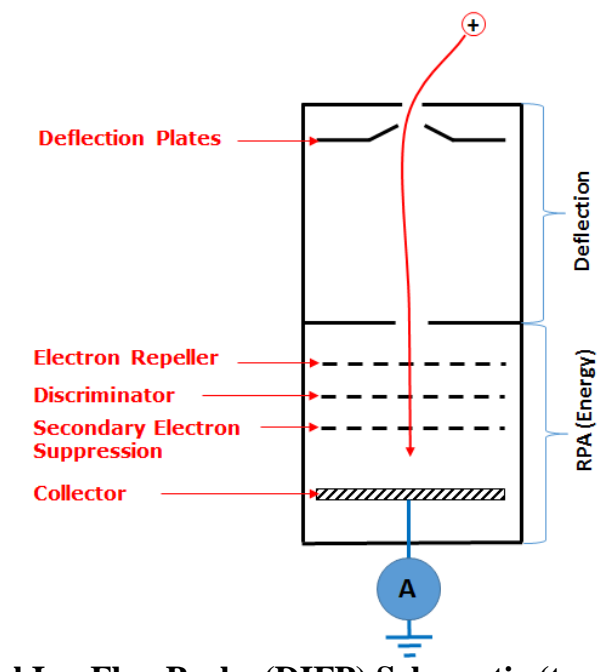

Figure 5. Differential Ion Flux Probe (DIFP) Schematic (top view at mid-plane)

\section{Tether Element (Biased Body)}

For a full scale electric sail, the tethers are tens of kilometers in length, and only tens of microns in diameter. Constructing a tether element that would fit inside the test chamber, as well as remain straight and fixed in a precise location, necessitated the creation of a custom test article instead of using a small piece of actual tether material. Figure 6 shows a picture of the tether element (also called the biased body) on the laboratory bench. The layout of the stainless steel tether element is shown in Table 3. To minimize end effects, a guarded design was employed for the tether element. The tether element assembly is mounted to a swing arm that allows the tether element to be moved out of the ion flow without breaking vacuum.

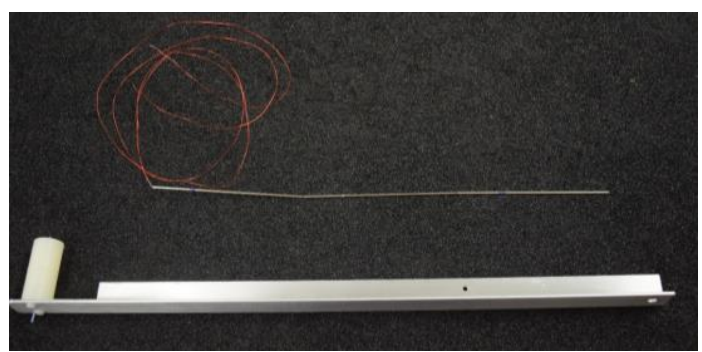

Figure 6. Stainless steel cylindrical tether element (top) and swing-arm support (bottom)

Table 2. Tether Element Layout

\begin{tabular}{|l|l|l|l|}
\hline Diameter & Section & Length & Role \\
\hline \multirow{3}{*}{$1.85 \mathrm{~mm}$} & Top & $9.7 \mathrm{~cm}$ & Guard (biased; no collection) \\
\cline { 2 - 4 } & Middle & $13.2 \mathrm{~cm}$ & Primary Element \\
\cline { 2 - 4 } & Bottom & $12.8 \mathrm{~cm}$ & Guard (biased; no collection) \\
\hline
\end{tabular}




\section{Vacuum Chamber}

The vacuum chamber was selected for the test based on three primary factors: 1) Its overall large size; 2) The high pumping speed of the vacuum system; and 3) The 2D motorized translation system in place. Key parameters of the vacuum chamber are shown in Table 3. Figure 7 shows the vacuum chamber interior layout.

Table 3. Vacuum Chamber Parameters

\begin{tabular}{llc}
\multicolumn{3}{c}{ Cylind rical Vacuum Chamber Parameters } \\
Property & Parameter (Units) & Typical \\
Chamber Length & L $(\mathrm{m})$ & 2.7 \\
Chamber Diameter & D (m) & 1.2 \\
Base Vacuum Pressure & P (Torr) & $3.0 \mathrm{E}-07$ \\
"Source On" Pressure & P1 (Torr) & $6.0 \mathrm{E}-06$ \\
Source to Tether Dist. & L1 $(\mathrm{m})$ & 1.0
\end{tabular}

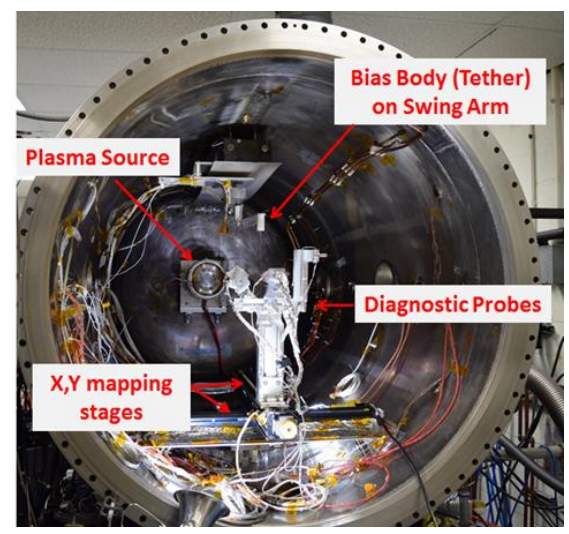

Figure 7. End view of vacuum test chamber

\section{Overall Arrangement}

The main components that make up the test system are arranged along the mid-plane of the chamber: The ion source is located at the eastern side; approximately $1 \mathrm{~m}$ downstream of the ion source is the tether element suspended vertically; and up to $300 \mathrm{~mm}$ downstream from the tether element is the probe array. The probe array is mounted to a 2D motor-driven translation stage that allows the probes to travel between $50 \mathrm{~mm}$ and $300 \mathrm{~mm}$ downstream of the tether element and $+/-100 \mathrm{~mm}$ along the chamber radius.

American Institute of Aeronautics and Astronautics 

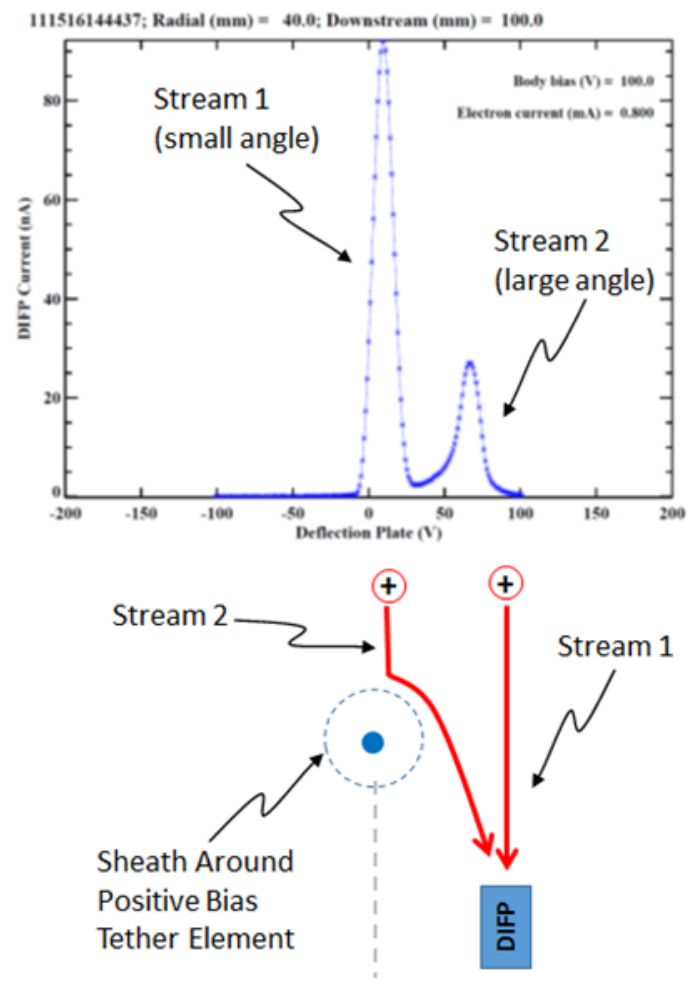
Figure 8. Top: Typical DIFP data (Current vs. Deflection Plate voltage); Bottom: Schematic diagram
correlated to data plot

\section{Ion Deflection and Plasma Sheath Measurements}

To develop a picture of the sheath thickness around the cylindrical tether element, and to verify the change in ion trajectories as they encounter the positively biased tether element, the DIFP is moved systematically along a range of downstream and radial locations. At each location, DIFP data such as shown in Figure 8 is acquired (Ion Current vs. Deflection Voltage). The plot in Figure 8 contains two peaks and represents the situation where a $+100 \mathrm{~V}$ bias is applied to the tether element (causing $100 \mathrm{eV}$ ions to be deflected or repelled). The tall peak occurs at low deflection plate voltage, which represents ions entering the DIFP at small angles. The short peak, however, occurs at a high deflection plate voltage, which means ions with high angle trajectories are present in the flow.

DIFP data sets gathered downstream from a positively biased tether element are always compared to freestream data where there is no bias on the tether element or the tether element is completely removed from the flow. Such comparisons show definitively the presence of a plasma sheath when there is a positive bias on the tether element, and show ions are deflected by the sheath into large angle trajectories.

Analyzing a set of DIFP data taken at multiple radial locations downstream of a positively biased tether element provides clear evidence the plasma sheath reflects ions that are flowing directly into the center of the sheath, and deflects ions that flow near the sheath edge. 


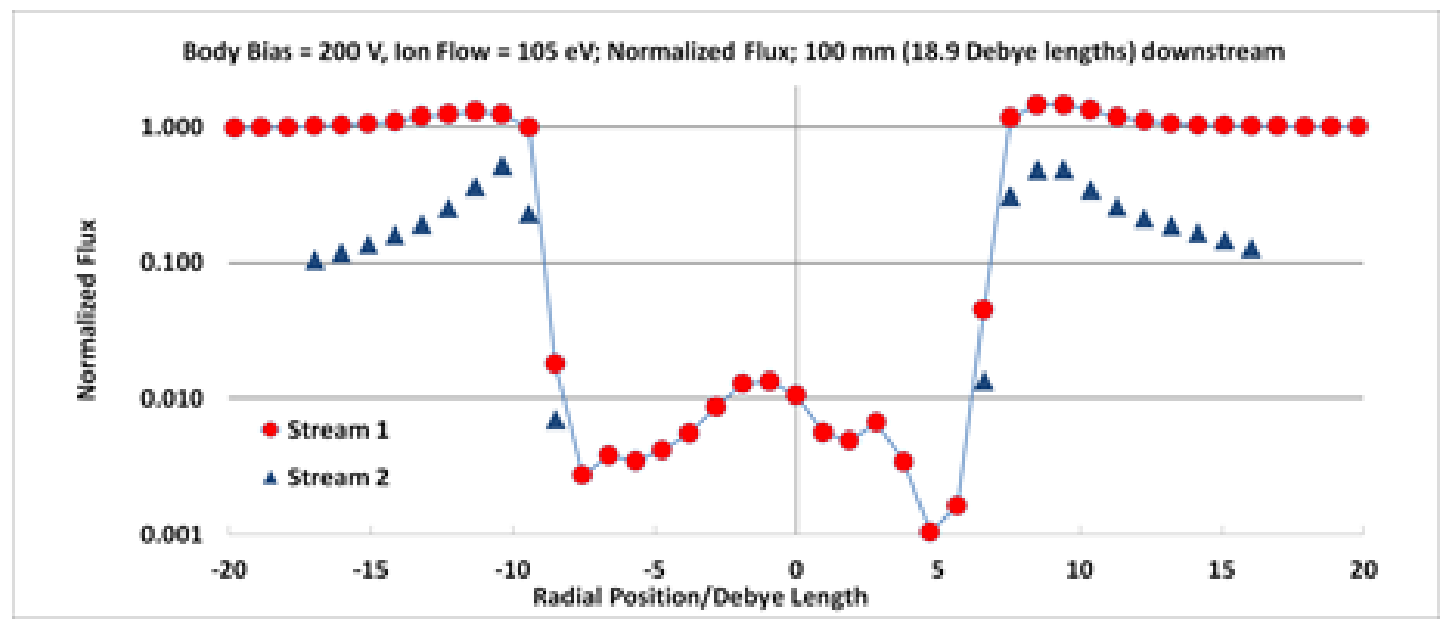

Figure 9. Normalized flux vs. Radial Position measured $100 \mathrm{~mm}$ downstream of the tether element $(+200 \mathrm{~V})$. The low flux region indicates the sheath around the tether is deflecting incoming particles - resulting in a void downstream of the tether element

Figure 9 shows the extent of the plasma sheath around a $+200 \mathrm{~V}$ tether element as $105 \mathrm{eV}$ ions drift into it. Both the small angle (stream 1) and large angle (stream 2) beams are impacted by the presence of the sheath. A low flux region (void) is created downstream of the sheath as the stream 1 particles are reflected and stream 2 particles are scattered into large angles. Data sets such as those plotted in Figure 10 help quantify the flow angles of particles scattered by the tether element sheath. Figure 10 represents just one downstream position, when flow angle data from other downstream positions is added, a comprehensive picture of the sheath can be constructed and compared to computer models.

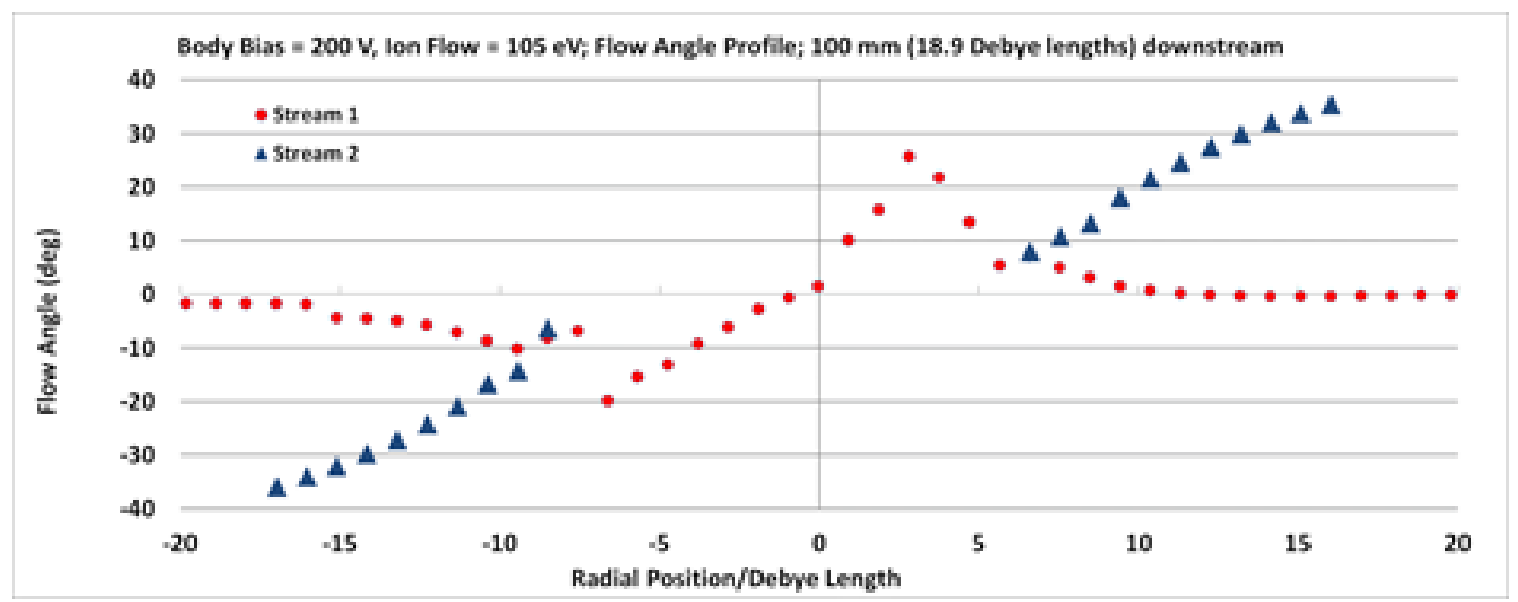

Figure 10. Flow angle vs. Radial Position measured $100 \mathrm{~mm}$ downstream of the tether element $(+200 \mathrm{~V})$. The sheath around the cylindrical tether element is deflecting/scattering ions into large angles with respect to the undisturbed flow. The extent of the sheath influence is $\sim 14$ Debye lengths $(\sim 74 \mathrm{~mm})$

\section{Electron Current Collection}

An important operational parameter for electric sails is the electron current collection that occurs as a result of the high voltage positive bias on each tether. The amount of electron collection will drive the total power required for the high voltage power supply and the emission requirements of the electron gun on the spacecraft. Therefore, throughout the test campaign to characterize the sheath effects associated with the positively biased tether element, current collection measurements were made.

A summary of the electron current measurements is provided in Figure 11. The data in Figure 11 cover a wide range of plasma conditions, tether bias voltages, and neutral pressures. The Mach numbers represent the ratio of ion drift speed to the ion sound speed. (Recall, ion sound speed is a function of electron temperature.) Interestingly, despite the wide range of conditions studied, the laboratory data tend to follow a common trend. Comparison of the

American Institute of Aeronautics and Astronautics 
laboratory electron collection data to the first generation Particle-In-Cell (PIC) code showed a significant under prediction by the code. The agreement is expected to improve as details of the plasma sheath in the PIC code are refined with data from DIFP measurements.

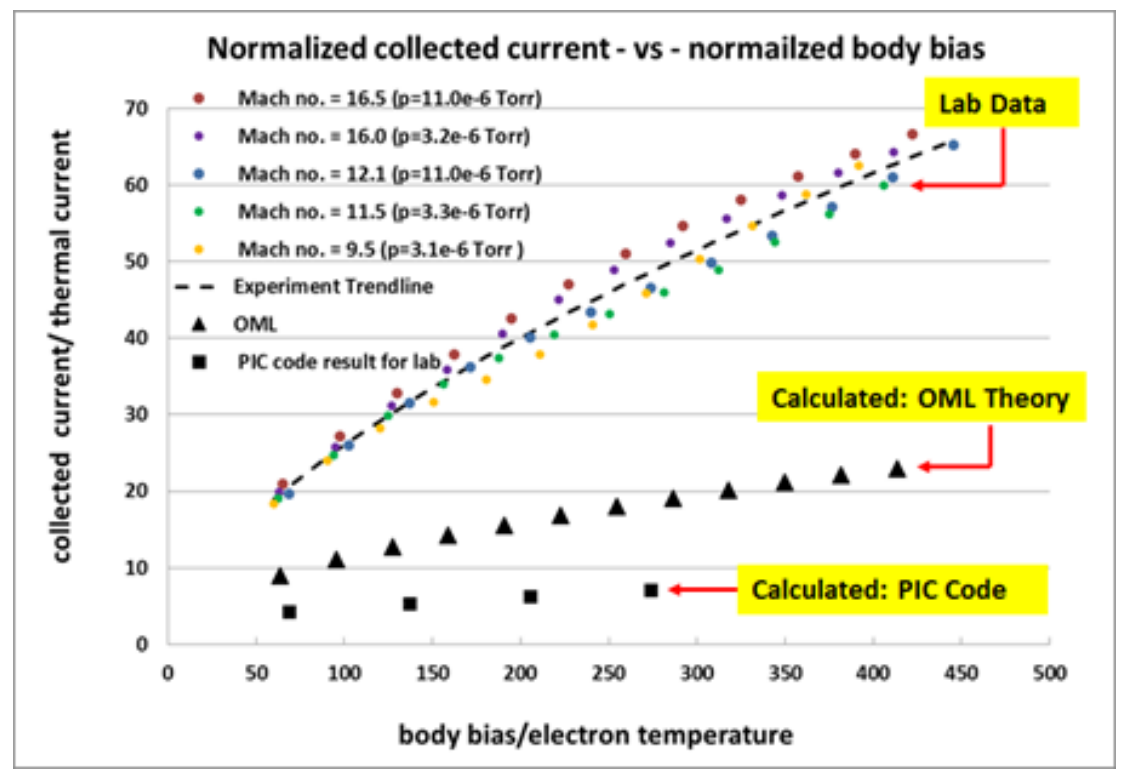

Figure 11. Tether element electron collection summary. The lab data remains grouped together, despite significant differences in ion speed (Mach number) and chamber neutral pressure. The Particle-In-Cell code was a first generation run with limits on the sheath scaling and resolution.

\section{E. Section Summary}

Electric sails are a relatively new concept for providing high speed propellant-less propulsion. Employing multiple tethers biased to high positive voltage levels $(\mathrm{kV})$, electric sails are designed to gain momentum from the solar wind by repelling solar wind protons. To maximize the area of the sail that interacts with the solar wind, electric sails rely on the formation of a large plasma sheath around each small diameter tether.

Motivated by interest in advancing the development of electric sails, a set of laboratory tests has been conducted to study the interaction of a drifting plasma with a sheath formed around a small diameter tether element biased at positive voltages. The laboratory test setup was created with Debye length scaling in mind to offer a path to extrapolate (via modeling) to full scale electric sail missions. Using an instrument known as a Differential Ion Flux Probe (DIFP) the interaction between a positively biased tether element and a drifting plasma has been measured for several scenarios. Clear evidence of the tether element sheath deflecting ions has been obtained. Maps of the flow angle downstream from the tether element have been made and they show the influence of the plasma sheath. Finally, electron current collection measurements have been made for a wide range of plasma conditions and tether element bias voltages. The electron collection data will have an impact on electric sail power requirements, as high voltage power supplies and electron guns will have to be sized to accommodate the electron currents collected by each tether.

\section{Mission Capture}

\section{F. Mission Design Constraints and Assumptions}

To demonstrate the value of electric sail propulsion, we have designed several missions that the design reference E-sail spacecraft can achieve. Although there are many classes of missions that the E-sail can achieve, here we concentrate on high solar inclination missions. High solar inclination missions are of major significance to the heliophysics community, as the study of the solar plasma emanating from the solar poles can potentially greatly advance current theories of how the sun works.

American Institute of Aeronautics and Astronautics 


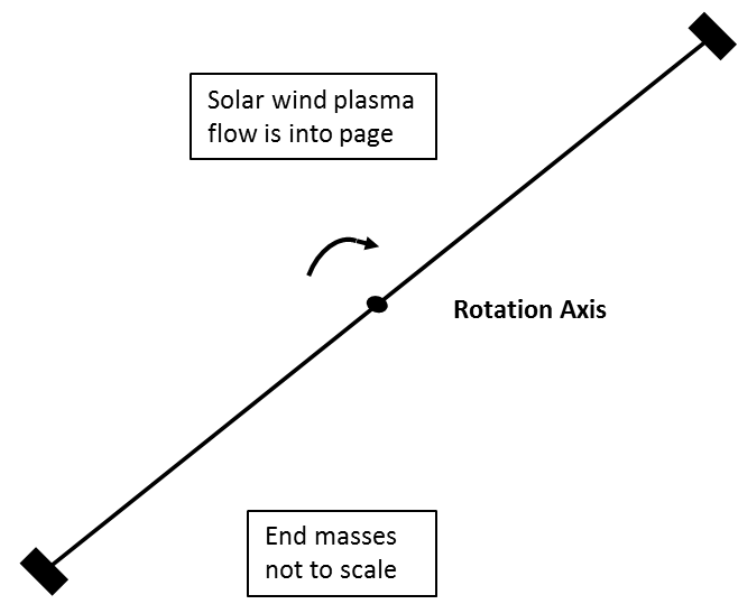

Figure 12. E-sail Spacecraft Pointed Directly at the Sun

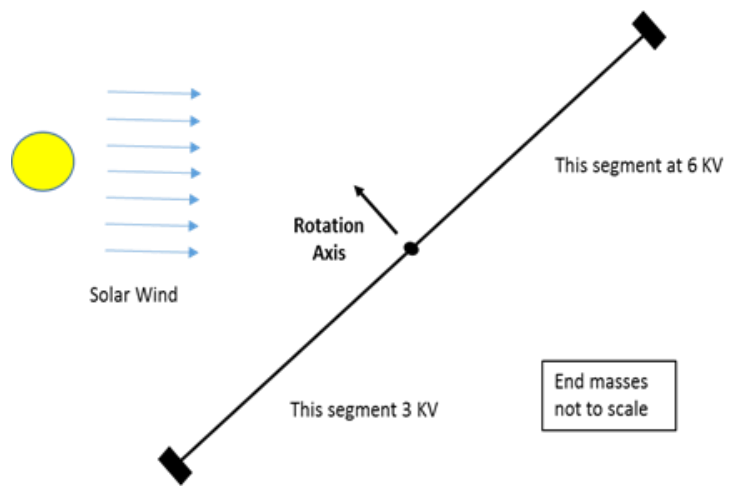

Figure 13. E-sail Tilt Actuated by Voltage Modulation

The E-sail thrust is maximized when the axis of rotation is pointed directly at the sun (see Figure 13). However, if the E-sail is only pointed directly at the sun, its usefulness is limited since the force will only be applied in the radial direction (the direction from the sun). In order to achieve a force in a direction other than radial, it is necessary to tilt the sail. This can be accomplished by creating a torque by varying the power on each half of the tether as it spins (see Figure 13). This will initiate a rotation rate in the desired direction to cause a tilt in the rotation axis of the E-sail with respect to the incident plasma. Partway through the rotation, the differential voltages as depicted in Figure 13 are swapped (i.e., the top segment in Figure 13 is $6 \mathrm{KV}$ and the lower segment $3 \mathrm{KV}$ ). This reverses the direction of the torque to stop the rotation.

\section{G. E-Sail Thrust Model for Mission Design}

The E-sail thrust changes as it tilts and changes the orientation of the tether to the incident plasma. The change in thrust with respect to this incident angle is a function of several factors, including a change in tether length exposed to the plasma, a reduction in the momentum transferred by the plasma to the tether, and the orientation of the tether around its rotation axis. Furthermore the interaction between incident protons and the electric field has uncertainties. In order to model uncertainties in these effects we have developed a parameterized E-sail thrust model that can easily be changed in the trajectory optimization tool MALTO. Such a parameter study will be conducted in the near future but is beyond the scope of this paper, so we have selected a nominal thrust model, which appears in the following equation.

$$
F m a g=F 0(\cos \theta / 2)^{1.9} r^{-1.16667}
$$

American Institute of Aeronautics and Astronautics 
In equation (2), Fmag is the resultant force, $\theta$ is the sail angle with respect to the incident plasma, and $\mathrm{r}$ is distance from the sun. F0 is the force on the E-sail produced at zero plasma incidence angle and $1 \mathrm{AU}$, and for the mission design studies appearing in this paper is 0.0144 Newtons. This is for a notional E-Sail TDM spacecraft that has a single tether that is $16,500 \mathrm{~m}$ in length. This force is also divided by the E-sail spacecraft mass of $24 \mathrm{~kg}$ to give a characteristic acceleration of $0.6 \mathrm{~mm} / \mathrm{sec}^{2}$. Characteristic acceleration is a metric borrowed from solar sails that allows a comparison of performance of various E-sail designs.

\section{High Solar Inclination Missions}

High solar inclination missions are challenging because orbital inclination change is quite costly. To go from a circular Earth orbit in the ecliptic plane to a solar polar orbit (an inclination change of $90 \mathrm{deg}$ ) requires approximately $30 \mathrm{~km} / \mathrm{s}$ of delta-velocity, which is immense. By contrast, the upper stage for the SLS EM-1 mission, a converted ULA Centaur upper stage, only does approximately $3 \mathrm{~km} / \mathrm{sec}$ to insert the Orion spacecraft into a lunar trajectory in a maneuver called the Trans Lunar Injection (TLI). The Centaur is one of the most powerful in-space conventional rockets available. This means that solar polar orbits are unreachable using current in-space propulsion capability. A solar polar orbit can be reached with a Jupiter gravity assist, which was accomplished by the Ulysses mission. The problem with using a Jovian gravity assist to achieve solar polar orbit is that it requires the spacecraft to remain a long distance from the sun while overflying the poles (this is a consequence of Jupiter's distance of 5.2 $\mathrm{AU}$ from the sun). An E-sail can achieve a solar polar orbit at a closer distance to the sun and with a lower time of flight than Ulysses.

A ground rule for the high solar inclination mission is that the E-sail remain within approximately $1 \mathrm{AU}$ of Earth to facilitate communication. (Since the design reference E-sail spacecraft is only $24 \mathrm{~kg}$, it can only carry a relatively small transmitter, meaning it needs to remain relatively close to Earth). Thus we constrain the semi-major axis of the achieved high inclination solar orbits to be $\sim 1 \mathrm{AU}$. Another ground rule is that the angle of the E-sail is constrained to be no more than 30 degrees from the incident solar plasma. This limitation is due to concerns about losing control of the E-sail at higher tilt angles.

With a characteristic acceleration of $0.6 \mathrm{~mm} / \mathrm{sec}^{2}$, the E-Sail TDM spacecraft can change inclination approximately 9.2 degrees per year. These results were generated by the simulation Copernicus using its finite thrust model subject to a semi-major axis constraint of $\sim 1 \mathrm{AU}$ (to facilitate communication with Earth), and with the sail set at a tilt angle of $30 \mathrm{deg}$. The sail is set at 30 degrees because this angle maximizes the component of thrust in the direction to change inclination and is as high as the ground rules allow (a slightly higher angle would likely have a greater thrust in the proper direction to change inclination). These results will be used to guide a full optimization study in the near future using MALTO and a more robust low thrust optimization simulation called S5.

\section{Deployment of Tethers and Initial Spinup of Large HERTS Spacecraft}

Of all the E-Sail systems trades performed by the team, the analyses of how to get all the tethers extended to their maximum length while getting the spacecraft initially spun up from being at rest to its required rotational rate at full tether deployment is the most challenging set of analyses of the various E-Sail trades performed to date.

The text which follows outlines the various spin up analyses that were performed on a notional HERTS spacecraft. This spacecraft had a total mass of $1000 \mathrm{~kg}$ and a total tether length of $2000 \mathrm{~km}$.

An electric sail (E-Sail) is a form of electrostatic spacecraft propulsion that deflects solar wind particles using many long, positively charged wires. The deflection of solar wind protons leads to a momentum exchange that propels the spacecraft. ${ }^{8}$ E-Sail spacecraft benefit from a smaller thrust drop off at large solar distances when compared to systems such as solar sails or solar electric thruster propulsion. The electrostatic sheath surrounding the charged tethers grows as the solar medium density decreases. This leads to a to decrease of roughly as $1 / r$ as the spacecraft moves away from the Sun as compared to $1 / r^{2}$ in the case of other forms of solar propulsion. ${ }^{9}$ For this reason, electric sails are a promising exploration tool for the outer solar system including investigations into the Heliopause.

Current designs for such spacecraft require the deployment of 20 to 100 individual tethers, each with a length measuring up to $20 \mathrm{~km}$, and carrying a positive voltage on the order of $25 \mathrm{kV}$. The thrust provided by the electric sail is directly proportional to the total length of tether, leading to a desire to increase tether length and number. These long tethers are held taut centrifugally, with the full system rotating at a low spin rate. Successfully deploying these long tethers is a difficult challenge as it requires a large amount of angular momentum and is affected complicated dynamics for these long, thin wires.

American Institute of Aeronautics and Astronautics 
Given the large distances covered by these long tethers, a large amount of angular momentum must be imparted into the system to allow it to reach its final configuration. An array of past space missions have utilized tethers for a variety of purposes, however all of these deployments have occurred for a single tether and have been assisted by the use of gravity gradient forces in low Earth orbit. The nearest analog to an E-Sail system deployment is the spin stabilization of solid rocket motor stages. However, these stages require orders of magnitude less angular momentum to be spun up due to their relatively small inertias.

For the specific case of electric sails, deployment of these tethers has in the past been studied by Janhunen, with current concepts requiring the use of remote units on the end of each tether. ${ }^{10}$ These remote units may be controlled either by reaction control or using a freely guided photonic blade. ${ }^{11}$ Fulton examined the deployment dynamics of an E-Sail, yielding solutions for the deployment time as well as torque requirements for a large electric sail deployment, further illustrating the difficulty of performing the required maneuvers. ${ }^{11}$

This work focuses on the practicality of imparting the required angular momentum into the E-Sail system to perform deployment and spin up of a full scale electric sail system. The following sections discuss the electric sail vehicle size studied, as well as assumptions about deployment mechanics and presents the deployment methods studied as well as the results obtained through analysis of these systems.

\section{H. Electric Sail Configuration Effects on Deployment}

The length of each tether and the number of tethers required has a significant effect on the momentum required to spin up and deploy the system. The trade between number of tethers and the length of each tether has been analyzed parametrically as a part of this study. For the purpose of the analysis here, a spacecraft with a mass of $1,000 \mathrm{~kg}$, featuring a total tether length of 2,000 km was assumed. Using the thrust calculated by Janhunen of 500 $\mathrm{nN} / \mathrm{m}$ at $1 \mathrm{AU}$ from the Sun, the total system yields a total thrust of $1 \mathrm{~N}$, and an acceleration of $1 \mathrm{~mm} / \mathrm{s}^{9} .{ }^{13} \mathrm{With}$ this level of acceleration, an E-Sail would be capable of reaching $100 \mathrm{AU}$ from the Sun (an area of interest for the Heliophysics community) within $10-15$ years. $^{14}$

The deployment is also heavily dependent on the mass per unit length of the tethers. Thin, ultrasonically welded tethers composed of one strand of $50 \mu \mathrm{m}$, and three strands of $25 \mu \mathrm{m}$ aluminum wire have been used for this analysis. Such a tether would have a mass per unit length of about $11.55 \mathrm{~g} / \mathrm{km}$ and a breaking strength of $100 \mathrm{mN}{ }^{15}$

Each tether is kept taut due the centrifugal force from the rotation of the spacecraft being greater than the thrust force on the tether itself. This stiffness factor ranges from 5 to 10 in the literature and a value of 5 has been adopted for this analysis, consistent with several E-Sail studies. ${ }^{15}$ For example, for a $20 \mathrm{~km}$ length of tether at $1 \mathrm{AU}$, the force on the tether due to the solar wind will be $10 \mathrm{mN}$. This yields the requirement that the tether be held at a tension of $50 \mathrm{mN}$ due to the rotation. This tension value drives the angular rate deployment value for the system.

For the purposes of this feasibility study, the dynamics of these long, thin tethers have been ignored. Friction in pulling the tethers off of the spool has also been omitted, and typically measures several milli-newtons at slow deployment velocities. Future work will be required to model these deployments as more complex systems, but the analyses performed here give a best case insight into the difficulties with deploying a large scale electric sail.

\section{Results for Several Deployment Options}

\section{A. Central Hub Forcing}

As a preliminary step, the deployment of the tethers from the central hub by giving the central hub an angular rate was studied. In this concept, thrusters mounted on the hub or on rigid booms away from the central hub provide the impulse to spin up the spacecraft. The tethers are released from the hub and extend due to centrifugal acceleration. This increases the inertia of the system, leading to a decreased angular rate by the conservation of angular momentum. Spinning up the spacecraft and deploying the tethers to their full length would require excessive spin rates in any configuration, so thrusting throughout deployment is required to maintain an angular rate in the hub.

This system assumes the use of a tether as described above, with the addition of a $10 \mathrm{~g}$ end mass placed on the end of each tether to help it remain taut during deployment. The length of each tether and the number of tethers has been studied for a range of configurations, summing to the total charged wire area of 2,000 km. For example, the nominal configuration studied by Janhunen consists of 100 tethers, each with a length of $20 \mathrm{~km}$.

The angular momentum required to spin up the spacecraft was studied for different numbers of tethers, and the results are shown in 4 . Control of this deployment was not considered as a part of this analysis, but would not have a significant effect on the integrated torque effort required.

American Institute of Aeronautics and Astronautics 


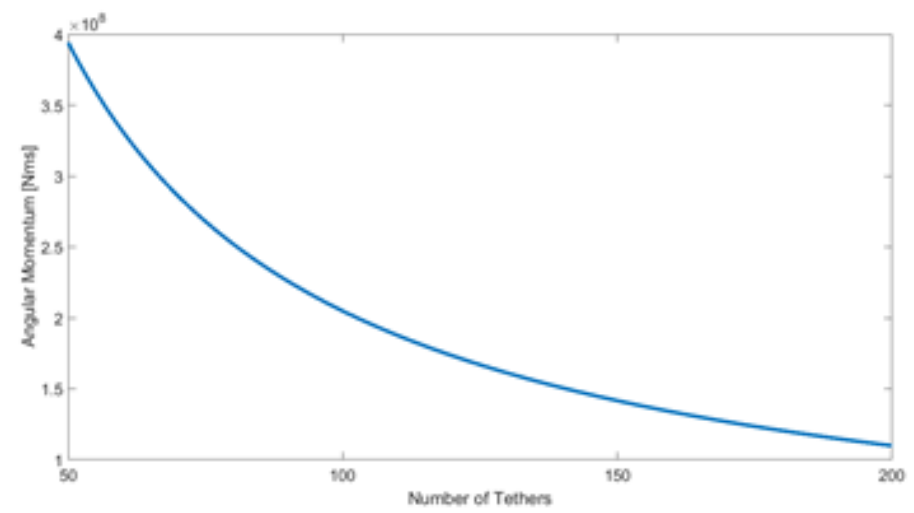

Figure 14. Momentum required to spin up the spacecraft to the required angular rate.

Since inertia grows as the square of distance, the momentum values are large despite the small mass of the tethers. Analysis shows that for most configurations achieving the desired acceleration levels, the total angular momentum of the system is on the order of $10^{8} \mathrm{Nms}$. These angular momentum values are many orders of magnitude larger than the capabilities of commercial reaction wheels.

With these large values for angular momentum required, it is impractical to spin up the spacecraft using thrusters placed on the body of the spacecraft (typically 1-2 $\mathrm{m}$ at most from the center of mass). Therefore, analysis has focused on the use of rigid booms to offset the thrusters a large distance from the center of mass $(5-20 \mathrm{~m})$. Placing a set of symmetric thrusters on booms at a distance from the hub, the thruster impulse required to spin up the full system was calculated and the results are shown in Figure 15. This calculation neglects the effect of decreasing spacecraft mass as propellant is burned, but this would yield only a small benefit since the inertia of the system is dominated by the tethers.

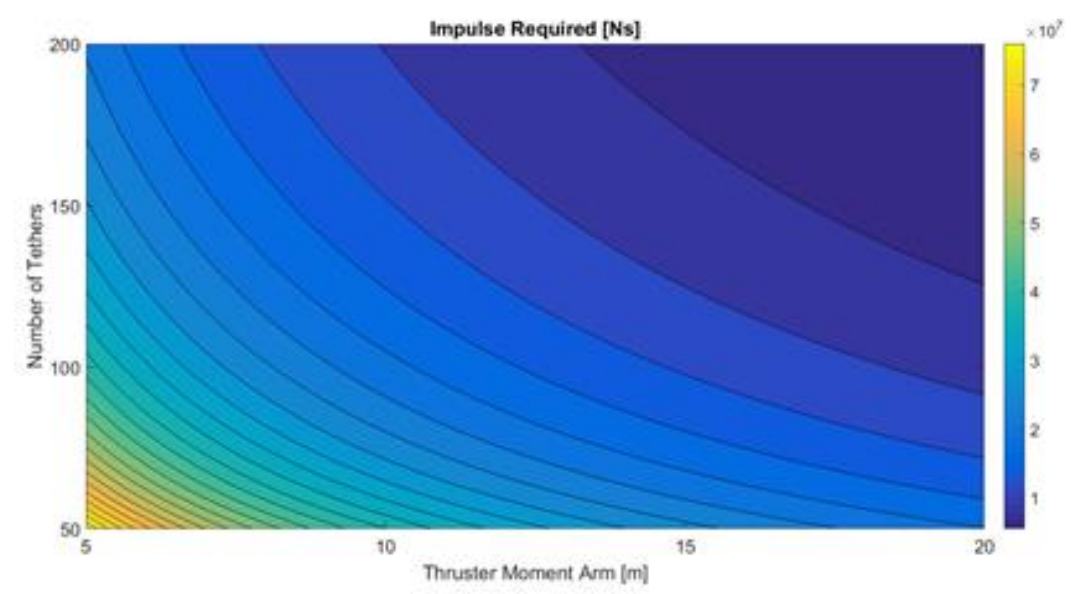

Figure 15. Impulse required to spin up the spacecraft to the required angular rate.

The values obtained for impulse were applied using the equation

$$
m_{\text {prop }}=\frac{J}{I_{s p} * g_{0}}
$$

Where: $J=$ impulse required in the system: $r=$ thruster moment arm; $I_{s p}=$ the specific impulse of the thruster; and $g_{0}=$ the gravitational acceleration on the surface of Earth.

A specific impulses of 3,000 s was chosen as an example case of an ion or hall thruster. A bipropellant hydrazine system was ruled out as early calculations showed no feasible designs for boom lengths less than $50 \mathrm{~m}$. 
All of these calculations neglect the increase in inertia from the propellant mass being placed at a distance from the. A basic analysis was performed to check this assumption and the resultant change in propellant mass was significantly less than $1 \%$ for all cases.

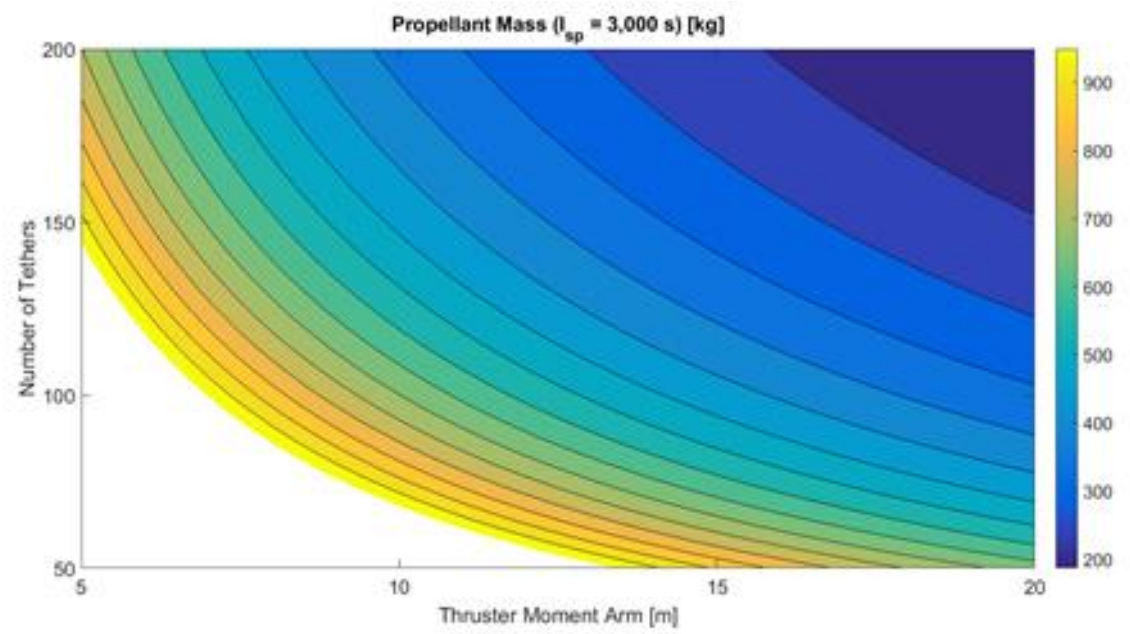

Figure 16. Propellant mass fraction required to spin up the $1,000 \mathrm{~kg}$ spacecraft to the required angular rate.

From these data, it is clear that spinning up the system using an ion or hall thruster is only feasible for large numbers of tethers with the thrusters placed on long booms away from the spacecraft center of mass. Boom lengths in excess of 10 meters would be required to allow a significant portion of the $1,000 \mathrm{~kg}$ spacecraft to be used for payload. An ion or hall thruster would be able to provide sufficient total impulse for a small subset of the configurations studied. However, such a system may run into limitations in deployment time due to the low level of thrust available. As a notional example, using two ion thrusters identical to those found on the Dawn spacecraft, with a thrust of approximately $100 \mathrm{mN}$ each, and a specific impulse of about 3,000 seconds, the burn time required to perform spin up was calculated for the same cases and can be seen in Figure 17.

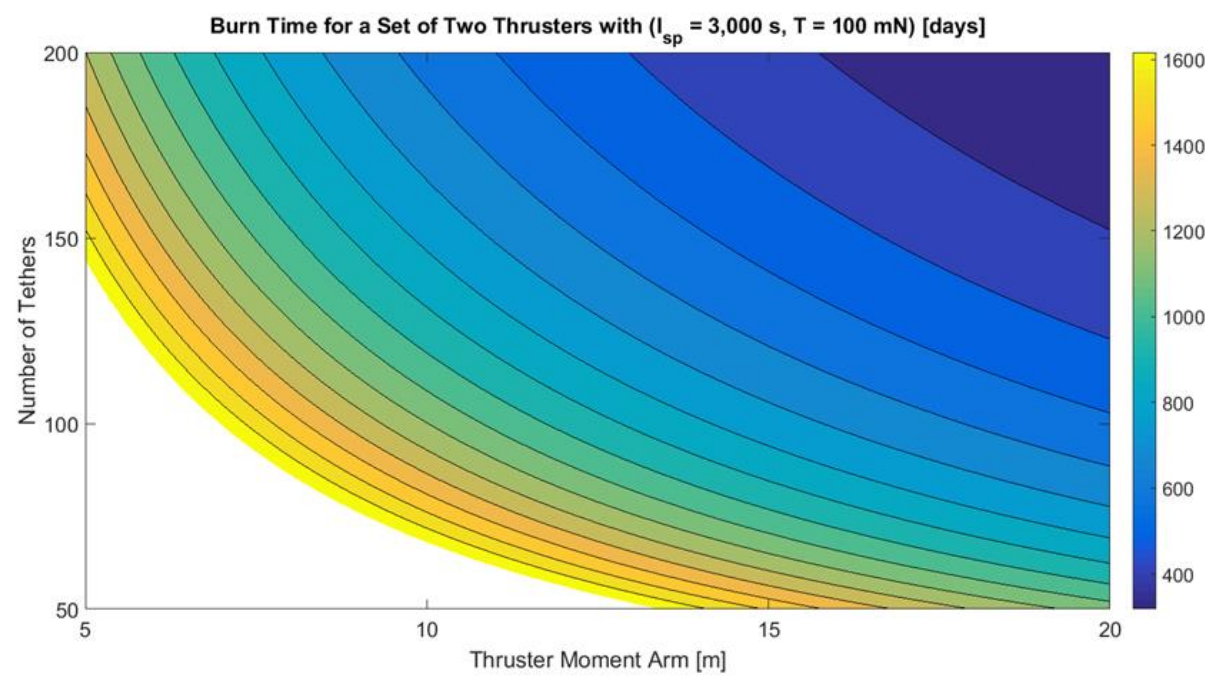

Figure 17. Burn time required for this low thrust system to perform spin up of the system.

These times range from 318 days for the $20 \mathrm{~m}$ moment arm, 200 tether case (top right of Figure 17), to a several years for most other configurations. Before taking into account controllability and tether tension constraints these times become a lower bound.

American Institute of Aeronautics and Astronautics 
Such a system would also require large solar arrays to provide power to the ion thrusters, requiring several kilowatts of power for each thruster. These large solar arrays would take up significant mass within the spacecraft.

\section{B. Propulsive End Units}

An alternative concept proposed by Janhunen uses a complement of propulsive units attached at the end of each tether to perform the deployment and then spin up of the full system. Such a system is advantageous compared to performing spin up at the hub, as it increases the moment arm of the thrusters by several orders of magnitude. It also has the potential to provide addition control throughout the course of the mission to maintain the spin rate in the presence of external forces. The main drawback of this system is the challenge presented by the complexity of operating 10's of individual modules which must effectively act as independent spacecraft. Reliability of such a system is a large concern, as a failure of one of the modules during deployment or spin up could have strong repercussions for the full E-Sail mission.

The propulsive end units were assumed to have a mass of $613 \mathrm{~g}$, in accordance with the values used by Janhunen in compiling an electric sail mass budget. An analysis was performed to determine the angular momentum of such a system, with the same requirements for tether tension as performed in Part A. The system has a larger inertia due to the increase in end mass when compared to the previously studied concept. However, the angular rate required to keep the tethers taut also decreases due to this end mass. The combination of these two factors yields an overall increase in angular momentum by roughly an order of magnitude.

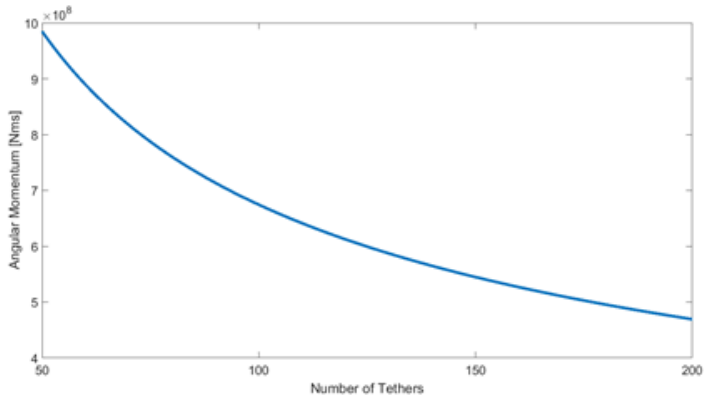

Figure 18. System angular momentum is greater using propulsive end units.

The impulse required to spin up the E-Sail was also calculated. Despite the larger angular momentum, the increase in moment arm from the previous example leads to a significant decrease in the required impulse, as seen clearly in Figure 19.

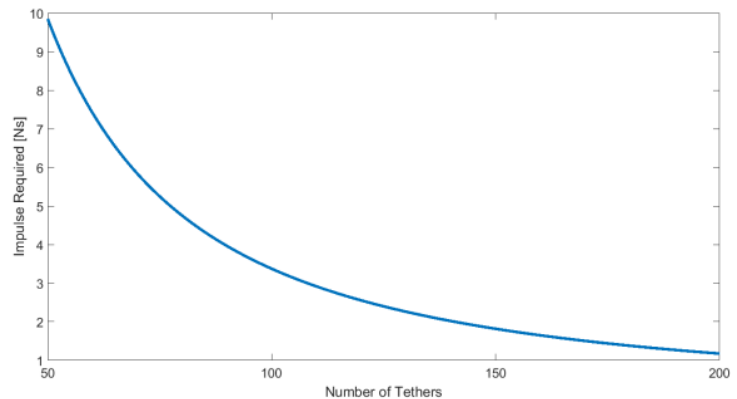

Figure 19. The impulse required decreases by several orders of magnitude when using propulsive end units due to the large moment arm. 


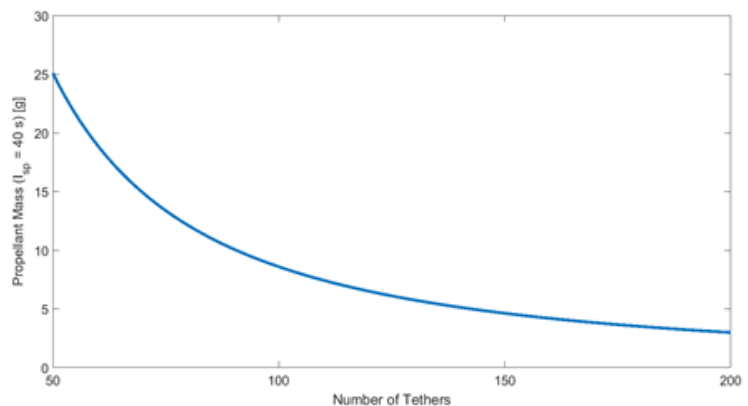

Figure 20. Propellant masses per end unit are well within the range of feasibility for such concepts.

\section{J. Section Conclusion}

It is clear from the data presented that deploying an electric sail without the use of propulsive end units is infeasible given the current state of the art in propulsion. These propulsive end units provide their own challenges with regard to complexity, but give large advantages in terms of propellant requirements.

\section{Conceptual Technology Demonstration Mission (TDM)}

\section{K. Technology Demonstration Mission Objectives}

Before any future Heliopause exploratory spacecraft or Interstellar spacecraft, which is to be propelled through space via an electric sail propulsion system is funded and built by any space fairing nation, the basic principles of propulsion via an electric sail propulsion system must first be demonstrated in deep space. The deployment of the required electric sail conductors must occur outside of the Earth's magnetosphere, since the electric sail produces thrust through the momentum exchange created by electrostatic repulsion that is present between the solar wind positively charged solar wind protons and a positively charged space conductor/s or tether/s. Therefore, our team believes that a Technology Demonstration Mission (TDM) must be developed and flown in deep space to prove the principles of electric sail propulsion.

Our proposed TDM mission concept (Figure 21) would utilize future NASA Space Launch System (SLS) ride share opportunities in the early 2020's with the TDM spacecraft being jettisoned from the SLS upper stage once the Orion Module safely has separated from the launch stack in the cis-lunar region of space. The overall focus of the TDM spacecraft investigation was to determine if all of the components necessary for an electric sail propelled TDM spacecraft could be packaged within the volume allocated for a single 12U spacecraft.

Before a representative electric sail TDM spacecraft could be conceptually developed, one key driving requirement (KDR) had to be set by the team, as this unique KDR set the overall systems subsystems design for the propulsion, power and thermal subsystems. This unique KDR is the characteristic acceleration value ay $1 \mathrm{AU}$.

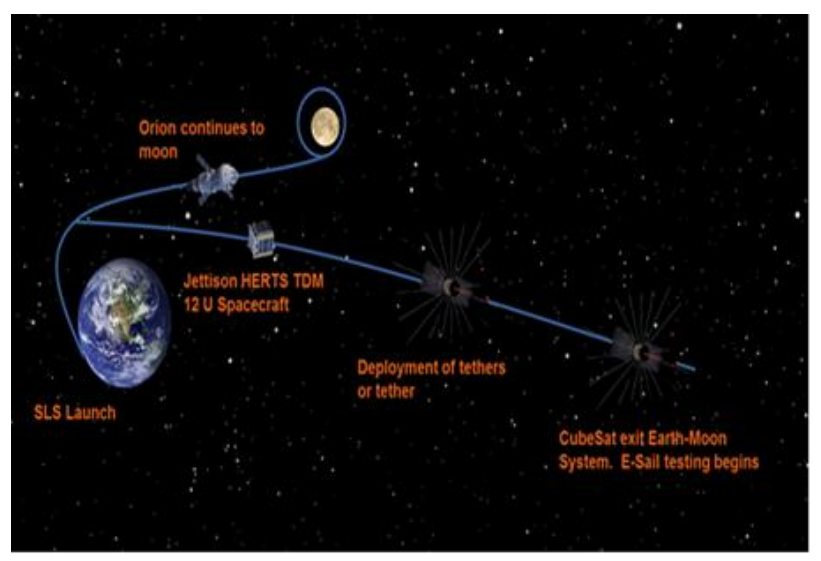

Figure 21. Overall Concept of Operation for the E-Sail Technology Demonstration Mission

American Institute of Aeronautics and Astronautics 
The chosen characteristic acceleration was set to be equal to, or greater than $0.6 \mathrm{~mm} / \mathrm{sec}^{2}$. This value was selected as it is an order of magnitude greater than the NEA Scout solar sail spacecraft characteristic acceleration value of $0.06 \mathrm{~mm} / \mathrm{sec}^{2}$ (Figure 22). It was our team's philosophy that this TDM shall demonstrate an acceleration potential that is close to the minimal characteristic acceleration required by the HERTS spacecraft, which is 1 $\mathrm{mm} / \mathrm{sec}^{2}$ as determined by the NASA HERTS team during the Phase I NIAC study. A comparison of characteristic accelerations of the NEA Scout to other spacecraft that have flown can also be seen in Figure 19.

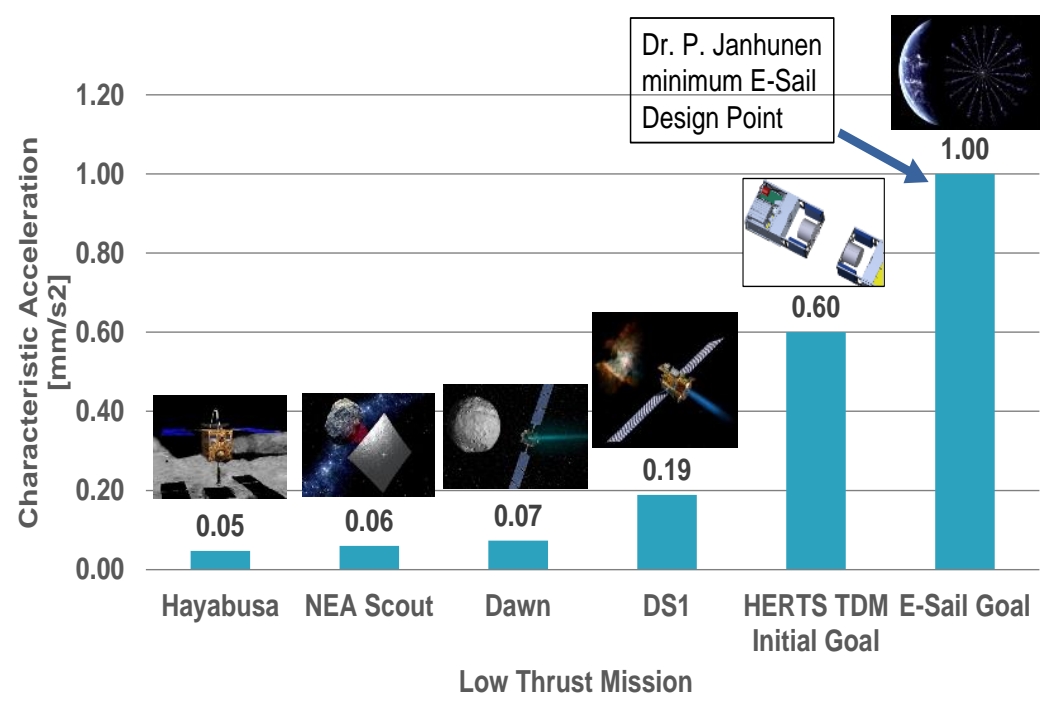

Figure 22. Comparison of E-Sail TDM Spacecraft Acceleration Rates to Other Spacecraft

The primary goals of the TDM as designed are to develop a CubeSat that can: 1) Deploy a 16,500 m conductive tether in deep space, 2) Charge the tether to a 6000V DC positive bias, 3) Accelerate the spacecraft, and 4) Steer the spacecraft. The secondary goal of the TDM is to collect meaningful science data either in route to a final destination or at the final destination.

\section{Science Obectives of a Technology Development Mission}

Our team has determined that the possible deep space locations and destinations for an electric sail Technology Demonstration Mission (TDM) are constrained by two spacecraft attributes, notably the electrical power production methodology and communication system architecture. Since this mission is to be a low risk mission, a decision was made that the electrical power system must use photovoltaic arrays as the source of electric power. Therefore, this decision limits the destination maximum range to $<5 \mathrm{AU}$ distances. The communication design requires electrical power to drive necessary spacecraft receivers and transponders. At a maximum range distance $>1 \mathrm{AU}$ total distance from the Earth, the physical demands (size mass, and power) of a communications system outgrow the space allocated for such a system in a $12 \mathrm{U}$ CubeSat architecture. Therefore, the team limited the reference maximum distance away from Earth to a 1 AU range.

Upon looking at these constraints the team determined that the best scientific returns would be from a mission that supported either: the Heliophysics science community or a mission that would support the planetary sciences, notable the asteroid scientists. One possible Heliophysics mission is a mission propelled by the E-Sail that takes Heliophysics sensors out of the solar system ecliptic plain to an inclination of $\sim 45$ degrees within 3 years. Figure 23 shows the effects of an electric sail spacecraft's initial characteristic acceleration versus the total inclination out of the solar systems ecliptic plain achieved over a three-year mission. This propulsion technology will enable small scientific spacecraft to get to many locations outside the ecliptic plain in order to investigate the sun and its affect upon space weather better. 


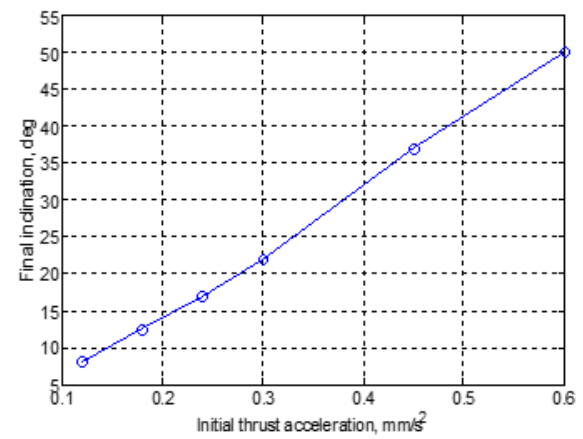

Figure 23. Inclination from Ecliptic Plane vs. Characteristic Acceleration over Three Year Trip

The other selected mission for a possible E-Sail TDM is one where the E-sail propelled spacecraft fly by numerous asteroids in a period of 3 to 5 years. The reference goal of asteroid flybys is a total of 10 per each TDM ESail spacecraft.

\section{TDM Spacecraft Concept}

The Marshall Space Flight Center's Advanced Concepts organization developed a concept of the TDM spacecraft. Since the mission must be conducted outside the Earth's geomagnetic field, the avionics and subsystems must be compatible with the radiation environment of deep space. Typically these components are more complex and expensive.

Another MSFC team in conjunction with JPL is developing the Near-Earth Asteroid (NEA) Scout vehicle which is a $6 \mathrm{U}$ CubeSat expected to fly on the first SLS mission EM-1. The NEA Scout is a solar sail demonstration flight that will travel to a near earth asteroid and survey the asteroid from a very close range. The NEA Scout spacecraft bus design offered a very close fit to the E-Sail TDM requirements. Therefore, the NEA Scout became the obvious point of departure for the bus design.

Shown in Figure 21 is a concept using the second flight of the SLS with a flight crew on-board. In this lunar bound flight, the spacecraft is ejected during the trans-lunar flight phase after the Orion crew capsule departs the SLS Upper Stage. Once the TDM is separated a safe range from the Orion module the spacecraft will begin the deployment of the $16,500 \mathrm{~m}$ of the tether. The spacecraft will then energize the conductor to a positive biased voltage of $600 \mathrm{~V} \mathrm{dc}$. It has been calculated that such a positive bias will create a Debye sheath of $\sim 30 \mathrm{~m}$ to $60 \mathrm{~m}$ in diameter around the energized conductor at a $1 \mathrm{AU}$ distance. This energized sheath will then electrostatically reflect and deflect the protons that occur in the solar wind thus producing thrust. Once the conductor is energized and thrust is produced, the testing of the E-Sail propulsion system will commence. Once initial testing is complete over a multiple day period, the spacecraft will begin to exit the ecliptic place to conduct the solar observations described in the Science Mission Objectives.

The function that the NEA Scout bus provides is a very close match to the E-Sail requirements. The primary issue yet to be address from the bus perspective is the communications requirements. The E-Sail TDM trajectory will be different than the NEA Scout, but the final trajectory has not been defined so no changes to the RF system have been addressed to date. JPL is developing the NEA Scout avionics hardware and the design is fairly mature. The flight is still several years away so modifications are possible. The E-Sail TDM assumed little of no changes to the NEA Scott for the preliminary concept. The hardware largely maps over one to one but the specific layout and positions of each subsystem differs in some degree due to the E-Sail propulsion interfaces and the use of two identical buses.

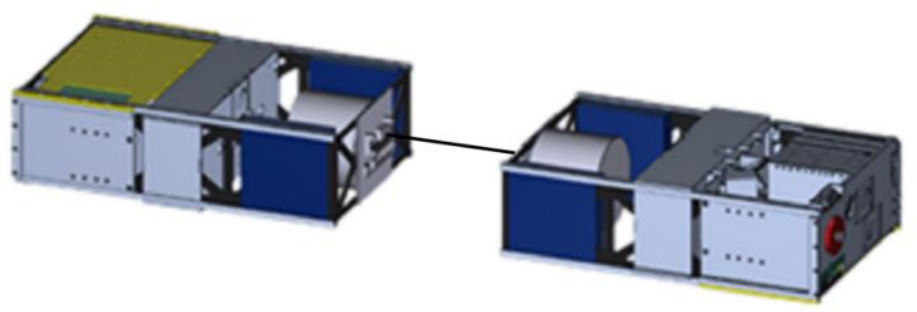

Figure 24. E-Sail TDM Spacecraft Configuration

American Institute of Aeronautics and Astronautics 
A preliminary layout of the conceptual E-Sail TDM spacecraft is shown in Figure 24, which reflects the NEA Scott heritage avionics and the E-Sail propulsion system as currently defined. The two identical spacecraft would be launch on top of each other and would be deployed for the SLS vehicle as an assembly. After deployment to acceptable distances from the SLS / Orion vehicle the two spacecraft separate and begin deploying the conductor.

\section{N. Future Work}

The electric sail work that our team is focused on is funded through September 2017, by the NASA Space Technology Mission Directorate (STMD). During these next months, a state of the art Particle-in-Cell (PIC) spacecraft model will be completed by a team member at the University of Alabama in Huntsville which will allow spacecraft designers the capability to deter-mine the effectiveness of an electric sail propulsion system for future spacecraft of various sizes. Upon completion of this model, our team will re-evaluate the spacecraft designs for both the Heliopause mission and the TDM mission.

In addition, our team has been given internal MSFC 2017 Technology Investment Program (TIP) funding to demonstrate the tether deployment from two 6U satellite mockups on the MSFC Flat Floor Facility during the summer of 2017. This work is fundamental to proving the functionality of various electric-sail subsystems, including the tether deployer. Many recent space tether missions have failed, because of various tether deployment issues. This flat floor testing will be one of the risk reduction steps our team is taking.

The team has also submitted a proposal to fly a tether deployer system on an upcoming 2018/2019 sub-orbital flight sponsored by NASA's STMD Flight Opportunities Program. The sub-orbital flights that are currently targeted in this solicitation may have up to 5 minutes of zero gravity time available. It is our team's belief that $200 \mathrm{~m}$ and possibly up to $1000 \mathrm{~m}$ of tether could be deployed in such a flight opportunity. However, the total amount of tether deployed is highly dependent upon the total zero gravity time, as well as the deployment speed of the as-built system. The lessons learned from the summer of 2017 flat floor testing will be integrated into the design of the suborbital flight hardware.

\section{O. Final Conclusions}

The fundamental research that is occurring at the MSFC in support of the Phase II NIAC project enumerated above will lead to a green, revolutionary propulsion system that may achieve travel speeds of up to 8-10 AU/yr. These spacecraft speeds are necessary for various future deep space missions of scientific discovery over the next 20 to 50 years. Before such elaborate missions can be fielded, an actual demonstration of this propulsion technology must be completed.

The authors believe such a TDM can be commenced when the SLS EM-2 flight is launched in 2021, and the TDM will be completed after three years of operations, at which time the reference spacecraft may achieve an inclination of 50 degrees out of our solar system's ecliptic plane.

At these locations, vast quantities of Heliophysics science data can be returned to scientists on Earth, thereby increasing our knowledge of the solar storms and resulting space weather phenomenon that eventually effect the Earth and many of the operational satellites in orbit about our planet.

\section{Acknowledgment}

The authors wish to acknowledge the NASA MSFC Advanced Concepts Office for their work in performing the concept studies for the HERTS TDM spacecraft.

We also wish to thank NASA's Space Technology Mission Directorate, notably the NASA Innovative Advanced Concepts (NIAC) Program, which is led by Dr. Jason Derleth for continuing to fund the Phase II HERTS NIAC project

\section{References}

1. Janhunen, P. and A. Sandroos, Simulation study of solar wind push on a charged wire: solar wind electric sail propulsion, Ann. Geophys., 25, 755-767, 2007

2. Mengali, G., A. Quarta and P. Janhunen, Electric sail performance analysis, J. Spacecr. Rockets, 45, 122-129, 2008 .

American Institute of Aeronautics and Astronautics 
3. Janhunen, P., The electric sail - a new propulsion method which may enable fast missions to the outer solar system, J. British Interpl. Soc., 61, 8, 322-325, 2008.

4. Quarta, A.A. and G. Mengali, Electric sail mission analysis for outer solar system exploration, J. Guid. Contr. Dyn., 33, 740-755, 2010.

5. Wiegmann, Bruce M., NASA Innovative Advanced Concepts (NIAC) Heliopause Electrostatic Rapid Transit System (HERTS) Phase II Proposal In response to: NNH15ZOA001N -15NIAC-A2, April 27, 2015.

6. Stone, N. H., Re-Thinking the use of the OML Model In Electric-Sail Development, Fifth International Conference on Tethers in Space, 24-26 May 2016, Ann Arbor, Michigan, USA.

7. Stone, N. H., Technique for measuring the differential ion flux vector, Rev. Sci. Instrum., 48, $1458,1977$.

8. P. Janhunen, "Electric sail, photonic sail and deorbiting applications of the freely guided photonic blade," Acta Astronautica, 2013.

9. P. Jahnunen, "Electric Sail for Spacecraft Propulsion," Journal of Propulsion and Power, 2004.

10. P. Toivanen and P. Janhunen, "Electric Solar Wind Sail: Deployment, Long-Term Dynamics, and Control Hardware Requirements," Advances in Solar Sailing, 2013.

11. J. Fulton and H. Schaub, "Sensitivity Analysis for the Electric Sail Deployment Dynamics Parameters," in 5th International Conference on Tethers in Space, Ann Arbor, Michigan, 2016.

12. P. Janhunen, "Status report of the electric sail in 2009," Acta Astronautica, vol. 68, pp. 567-570, 2010.

13. G. Mengali, A. A. Quarta and P. Janhunen, "Considerations of Electric Sailcraft Trajectory Design," in Fifth IAA Symposium on Realistic Near-Term Advanced Scientific Space Missions, Aosta, Italy, 2007.

14. B. M. Wiegmann, "Heliopause Electrostatic Rapid Transit System (HERTS)," 2015. [Online]. Available: http://ntrs.nasa.gov/archive/nasa/casi.ntrs.nasa.gov/20150016542.pdf.

15. P. Janhunen, A. Quarta and G. Mengali, "Electric solar wind sail mass budget model," 2013. [Online]. Available: http://www.geosci-instrum-method-data-syst.net/2/85/2013/gi-2-85-2013.pdf.

American Institute of Aeronautics and Astronautics 